\title{
RESOLUTION AND KINEMATICS OF MOLECULAR GAS SURROUNDING THE CLOVERLEAF QUASAR AT $z=2.6$ USING THE GRAVITATIONAL LENS
}

\author{
M. S. Yun, ${ }^{1}$ N. Z. Scoville, J. J. Carrasco, and R. D. Blandford \\ California Institute of Technology, Pasadena, CA 91125 \\ Received 1996 December 4; accepted 1997 January 23
}

\begin{abstract}
Gravitational lenses have long been advertised as primitive telescopes, capable of magnifying cosmologically distant sources. In this Letter we present new, 0."9 resolution CO (7-6) observations of the $z=2.56$ Cloverleaf quasar (H1413+117) and spatially resolved images. By modeling the gravitational lens, we infer a size scale of 0.3 $(\sim 1 \mathrm{kpc})$ for the molecular gas structure surrounding the quasar, and the gas has a kinematic structure roughly consistent with a rotating disk. The observed properties of the $\mathrm{CO}$-emitting gas are similar to the nuclear starburst complexes found in the infrared luminous galaxies in the local universe, and metal enrichment by vigorous star formation within this massive nuclear gas complex can explain the abundance of carbon and oxygen in the interstellar medium of this system observed when the universe was only a few billion years old. Obtaining corresponding details in an unlensed object at similar distances would be well beyond the reach of current instruments, and this study highlights the less exploited yet powerful use of a gravitational lens as a natural telescope.

Subject headings: cosmology: observations — galaxies: starburst — infrared: galaxies -
\end{abstract} ISM: molecules - quasars: individual (H1413+117)

\section{INTRODUCTION}

The Cloverleaf quasar $(\mathrm{H} 1413+117)$ is one the most distant objects in which the presence of a large amount of cold, molecular gas is inferred from the detection of bright $\mathrm{CO}$ emission (Barvainis et al. 1994). Its spectral energy distribution peaks in the far-infrared as in another luminous highredshift object, FSC 10214+4724 $(z=2.29)$ (Barvainis, Antonucci, \& Coleman 1992; Rowan-Robinson et al. 1993; Eisenhardt et al. 1996), and the total infrared luminosity and inferred molecular gas mass are comparable for these two objects. The optical image consists of four clearly separated peaks, implying that the (unseen) lens lies close to the line of sight to the quasar (Magain et al. 1988; Kayser et al. 1990). While the optical quasar may appear unresolved, the surrounding gas and dust are expected to be substantially extended, and the new $\mathrm{CO}$ (7-6) observations are made at 1" resolution in order to resolve the distribution of the molecular gas complex surrounding the quasar. Previous CO observations at much lower spatial resolutions $\left(\theta=3^{\prime \prime}-8^{\prime \prime}\right)$ had failed to resolve the molecular gas structure (Barvainis et al. 1994; Wilner, Zhao, \& Ho 1995).

In the following sections we show that the $\mathrm{CO}(7-6)$ emission in the Cloverleaf quasar is indeed resolved by our new interferometric observations and demonstrate that 0.1 scale spatial details can be revealed by gravitational lensing in addition to the usual luminosity amplification. This magnifying property of gravitational lenses holds promise for probing minute details of distant objects unachievable through conventional observational approaches.

\footnotetext{
${ }^{1}$ Present address: National Radio Astronomy Observatory, P.O. Box 0, Soccoro, NM 87801; myun@nrao.edu.
}

\section{OBSERVATIONS AND RESULTS}

Aperture synthesis observations of $\mathrm{CO}(7-6)$ emission $\left(\nu_{0}=\right.$ $806.651776 \mathrm{GHz}$ ) redshifted to $226.70 \mathrm{GHz}$ were carried out at the Owens Valley Millimeter Array between 1994 December and 1995 March in the equatorial and high-resolution configurations with longest baselines $200 \mathrm{~m}$ (east-west) and $220 \mathrm{~m}$ (north-south). Typical system temperatures were 500-800 K (single sideband), mainly contributed by the atmosphere. A digital correlator configured with $120 \times 4 \mathrm{MHz}\left(5.3 \mathrm{~km} \mathrm{~s}^{-1}\right)$ channels covered a total velocity range of $636 \mathrm{~km} \mathrm{~s}^{-1}$. The data were calibrated using the standard Owens Valley Millimeter Array program (MMA; Scoville et al. 1992), and the continuum and spectral line maps are made with DIFMAP (Shepherd, Pearson, \& Taylor 1994). Data from a total of 30 baseline pairs cover the inner $12-180 \mathrm{k} \lambda(u, v)$-plane, and the resulting synthesized beam is $0.86 \times 1.0\left(\mathrm{P} . \mathrm{A} .=73^{\circ}\right)$ with natural weighting of the visibility data. The $(u, v)$-coverage is quite uniform, and the synthesized beam is well behaved; the largest sidelobes appear $2^{\prime \prime}$ north and south of the main beam with $20 \%$ of the peak amplitude. The CLEANing of the maps was limited only to the central $3^{\prime \prime}$ diameter region; no emission is seen elsewhere in the "dirty" maps. The positional accuracy of the resulting maps is better than 0"2. After $25 \mathrm{hr}$ of on-source integration, we achieved 3.2 and $4.9 \mathrm{mJy}^{\text {beam }}{ }^{-1}$ sensitivity in the upper and lower sidebands of the $1 \mathrm{GHz}$ bandwidth analog correlator continuum data. The $\mathrm{CO}$ (7-6) emission appears in only half of the total 120 spectrometer channels, and $1 \sigma$ noise in each $110 \mathrm{MHz}\left(\Delta V=145 \mathrm{~km} \mathrm{~s}^{-1}\right)$ averaged channel maps (Figs. $1 c$ and $1 d$ ) is $8.3 \mathrm{mJy} \mathrm{beam}^{-1}$. No continuum emission is detected in the $230 \mathrm{GHz}$ USB continuum data, and the $3 \sigma$ upper limit of $15 \mathrm{mJy}^{\text {beam }}{ }^{-1}$ is marginally consistent with the $240 \mathrm{GHz}$ continuum flux of $18 \pm 2$ mJy reported by Barvainis et al. (1995). A summary of the observations and results is given in Table 1.

The new CO (7-6) map resolves the source for the first time 

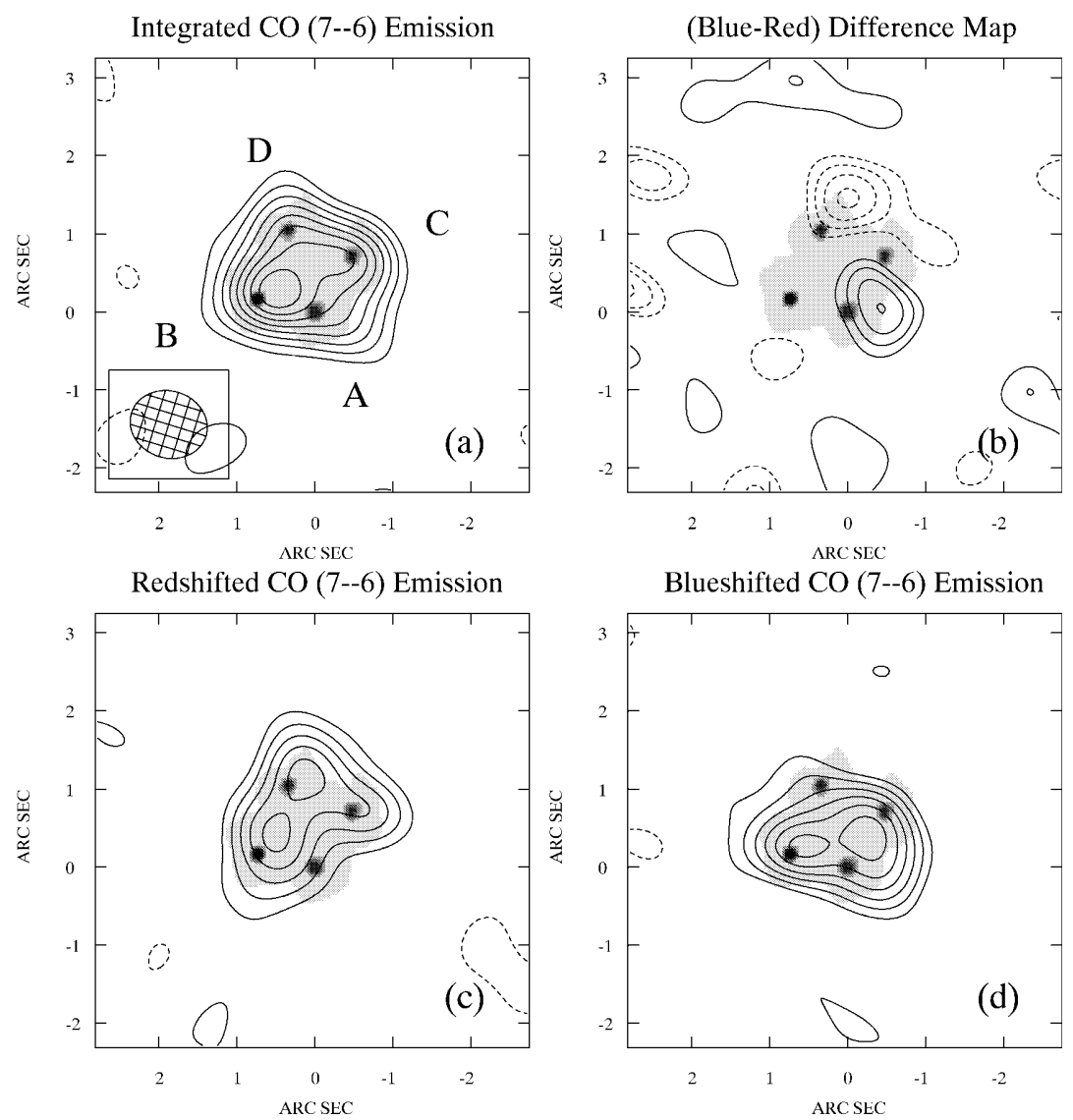

FIG. 1.-(a) Velocity-integrated map of CO (7-6) emission from the Cloverleaf quasar $(\mathrm{H} 1413+117)$ at $0.86 \times 11^{\prime \prime} 0\left(\mathrm{P} . \mathrm{A} .=73^{\circ}\right)$ resolution in contours overlaid on the gray-scale optical image obtained with the Hubble Space Telescope (Falco 1993). The contours are $-3,-2,2,3,4,5,6,7,8$, and 9 times the rms noise (5.3 $\mathrm{mJy}_{\text {beam }}{ }^{-1}$ ). Image A is brightest in optical light, but the CO peaks near image B, probably reflecting real differences in the underlying distributions. The coordinates are in offset with respect to image A. (b) A difference map between the redshifted and blueshifted emission (shown in $[c]$ and $[d$, respectively]) made in order to test whether the two velocity components are resolved. The contour levels are the same as in $(c)$ and $(d)$ for a direct comparison. $(c, d)$ Blueshifted and redshifted CO (7-6) emission averaged over $145 \mathrm{~km} \mathrm{~s}^{-1}$ distinctly different shown in contours superposed on the HST image. The contours are $-3,-2,2,3,4,5$, and 6 times the rms noise $\left(8.3 \mathrm{mJy}_{\text {beam }}{ }^{-1}\right)$. The blue and red images consist of two or three bright peaks, each with a distribution distinctly different from the other's and from the optical light. While the apparent difference in morphology between the blue and red images is striking, only $30 \%$ of the total flux remains in the difference map, suggesting that the bulk of the flux originates very close to the quasar.

(Fig. 1). The velocity-integrated CO emission (Fig. 1a) approximately follows the optical light, suggesting that the $\mathrm{CO}$ source is close to the quasar. However, the peak of the $\mathrm{CO}$ emission is located near image $\mathrm{B}$, whereas image $\mathrm{A}$ is the brightest in the optical and infrared-perhaps a first hint that the distri-

TABLE 1

SUMMARY OF THE OBSERVATIONS

\begin{tabular}{|c|c|}
\hline Parameter & Value \\
\hline R.A. $(B 1950) \ldots \ldots \ldots \ldots \ldots$ & $14^{\mathrm{h}} 13^{\mathrm{m}} 20.08$ \\
\hline Decl. $(\mathrm{B} 1950) \ldots \ldots \ldots \ldots \ldots$ & $11^{\circ} 43^{\prime} 37^{\prime \prime} 8$ \\
\hline$\nu_{\mathrm{obs}}, \ldots+\cdots, \cdots, \cdots, \cdots$ & $226.7035 \mathrm{GHz}$ \\
\hline$\left\langle z_{\mathrm{CO}(7-6)}\right\rangle \ldots \ldots \ldots \ldots \ldots \ldots$ & $2.5582 \pm 0.0003$ \\
\hline Luminosity distance, $D_{L}{ }^{\mathrm{a}} \ldots \ldots$ & $10.2 h^{-1} \mathrm{Gpc}$ \\
\hline Angular size distance, $D_{A}{ }^{\mathrm{b}} \ldots$ & $\begin{array}{c}0.79 h^{-1} \mathrm{Gpc} \\
\left(1^{\prime \prime}=3.8 h^{-1} \mathrm{kpc}\right)\end{array}$ \\
\hline$\theta_{\text {FWнм }}+\ldots \ldots \ldots \ldots \ldots \ldots$ & $0.86 \times 1 . " 0\left(\right.$ P.A. $\left.=73^{\circ}\right)$ \\
\hline$(\Delta V)_{\text {FWHM }}+\cdots \cdots \cdots \cdots \cdots$ & $339 \pm 21 \mathrm{~km} \mathrm{~s}^{-1}$ \\
\hline$S_{\mathrm{CO}} \Delta V \ldots \ldots+\cdots, \ldots, \ldots$ & $41 \pm 4 \mathrm{Jy} \mathrm{km} \mathrm{s}{ }^{-1}$ \\
\hline$L_{\mathrm{CO}}(7-6)\left(=4 \pi D_{L}^{2} S \Delta V \nu_{\mathrm{obs}}\right) \ldots$ & $5.5 \times 10^{8} h^{-1} L_{\odot}$ \\
\hline$M_{\mathrm{H}_{2}} \ldots \ldots \ldots \ldots \ldots \ldots$ & $2.5 \times 10^{10} h^{-2} M_{\odot}($ for $\mu=10)$ \\
\hline$M_{\mathrm{dyn}} \ldots \ldots \ldots \ldots \ldots \ldots \ldots \ldots \ldots \ldots \ldots \ldots \ldots \ldots$ & $2.6 \times 10^{10}[R(\mathrm{kpc})]^{-1}(\sin i)^{-2} M_{\odot}$ \\
\hline
\end{tabular}

bution of the $\mathrm{CO}$ emission is different from that of the quasar light. Since the $\mathrm{CO}$ emission does not form an Einstein ring (cf. MG 1131+0456; Hewitt et al. 1988), the CO-emitting region must be smaller in size than that of the tangential caustic, $\sim 1^{\prime \prime}$ or about $4 h^{-1} \mathrm{kpc}$ (assuming that $\Omega_{0}=1$ ). Our integrated $\mathrm{CO}(7-6)$ spectrum has the same profile as the IRAM $30 \mathrm{~m}$ spectrum (R. Barvainis 1996, private communication), and it is not shown because of space limitations.

In order to study the kinematics of the gas, we produced separate images of the blueshifted and redshifted gas (Figs. 1c and $1 d$ ). Clear differences from the optical image are seen in both the blueshifted and the redshifted images, while the difference between the two is also significant, as shown in Figure $1 b$. Thus two kinematically distinct sources have been spatially resolved by the gravitational lens. As in the velocityintegrated image (Fig. 1a), the blueshifted and redshifted gas images also have multiple bright peaks, but they are spatially displaced from each other and from the optical quasar positions. This displacement is quite significant, each $\sim 7$ times the rms noise in the maps. Because the position information depends on the phase of the visibilities, which is measured much more accurately than amplitude by an interferometer, the significance of the offset is even greater. In addition, the 


\section{Lens Model Potential $\quad$ Redshifted CO Source Model Btueshifted CO Source Model}

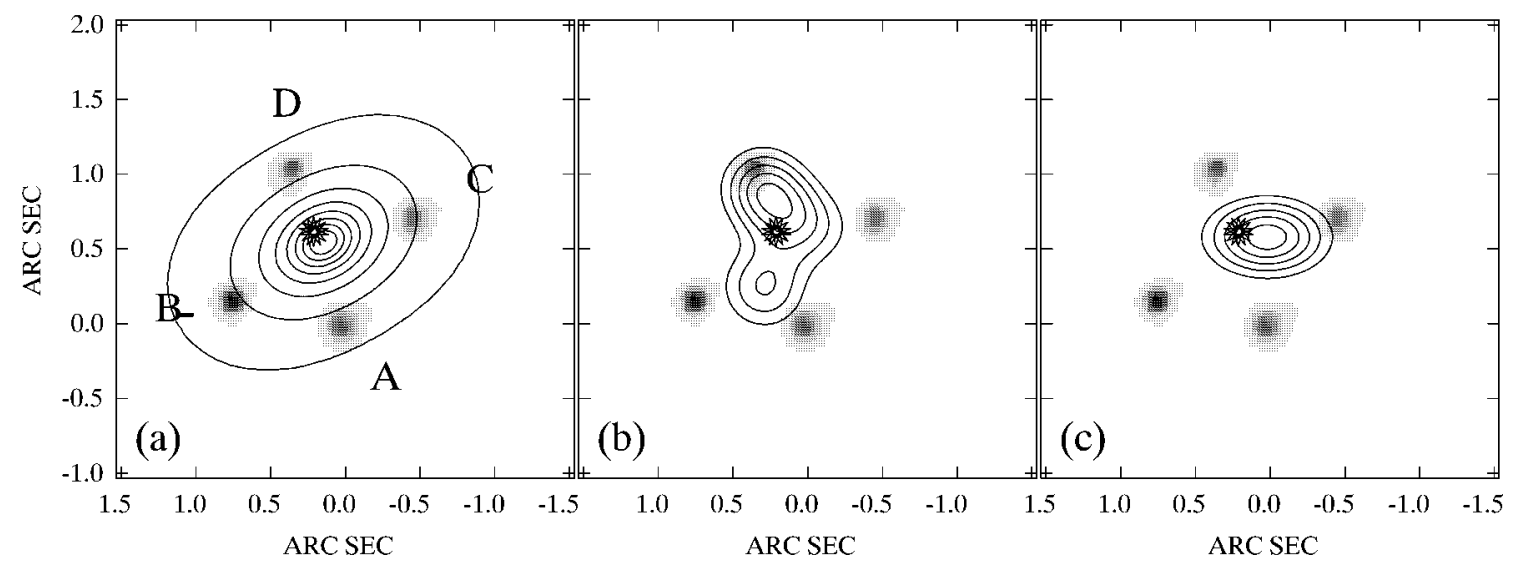

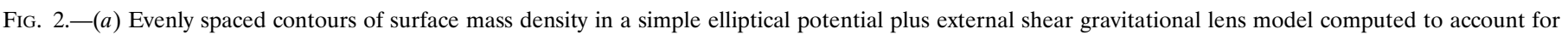

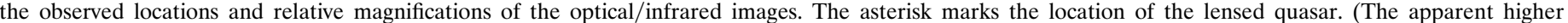

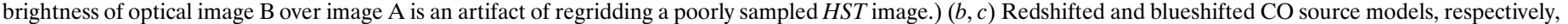

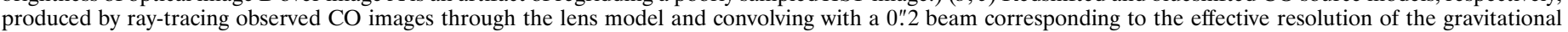

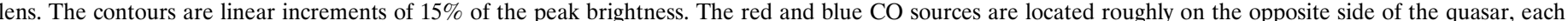

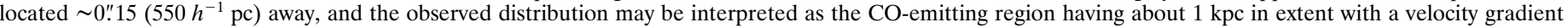
like that of a rotating disk.

fact that the multiple peaks in both blueshifted and redshifted $\mathrm{CO}$ images can be mapped back to single distinct (but different) features in the source plane further supports the reality of observed differences (see below).

Comparison of our data with unpublished Plateau de Bure $(\mathrm{PdB})$ interferometer maps ( $\mathrm{R}$. Antonucci \& R. Barvainis 1996, private communication) also lends support to the extended nature of the $\mathrm{CO}$ emission. The higher resolution $\mathrm{PdB}$ maps show a three-image geometry for the $\mathrm{CO}$ emission, similar to our own, but observed differences as a function of velocity are much less pronounced. The total line flux mapped by the PdB interferometer is about $25 \%$ less than our measurement, and their $(u, v)$-coverage poorly samples the baselines shorter than $50 \mathrm{~m}$ in length (needed to map 2"-3" scale structures). Only $30 \%$ of the total flux is seen in the difference map (Fig. $1 b$ ), and thus one may infer that about $70 \%$ of the line flux originates in a very compact region, very close to the quasar, while the remaining $30 \%$ lies farther out, if both observations are accurate.

\section{GRAVITATIONAL LENSING MODEL AND INFERENCES}

In order to test our hypothesis that the observed difference between the blue and red $\mathrm{CO}$ images is due to spatial resolution of the gas distribution and kinematics, we have developed a gravitational lens model. We have modeled the lens as a single elliptical potential with an external shear (Schneider, Ehlers, \& Falco 1992)

$$
\begin{aligned}
\psi= & b\left[1+\left(1-\gamma_{c}\right)(x / s)^{2}-2 \gamma_{s}\left(x y / s^{2}\right)\right. \\
& \left.+\left(1+\gamma_{c}\right)(y / s)^{2}\right]^{q}-\gamma_{1}\left(x^{2}-y^{2}\right)-2 \gamma_{2} x y
\end{aligned}
$$

where $b$ measures the size of the Einstein ring and $s$ is a core radius. The parameters $\gamma_{c}$ and $\gamma_{s}$ represent the ellipticity in the galaxy potential, and the parameters $\gamma_{1}$ and $\gamma_{2}$ measure the strength of the tidal action of external galaxies. The exponent $q$ is dictated by the radial density profile. A value $q=0.5$ corresponds to the (pseudo)isothermal case. We solved for parameters which reproduce the HST image locations (Falco
1993) and which also recover the three relative magnifications of the four $K$-band images measured nearly contemporaneously with the Keck telescope (M. Pahre 1996, private communication). These relative magnifications are quite similar to their optical counterparts. We take this as an indication that microlensing is unimportant. For an emitted wavelength $\sim 600$ $\mathrm{nm}$, a thermal source would be too large to be treated as a point source when microlensed by $\sim 0.1 M_{\odot}$ stars in an intervening galaxy (cf. Rauch \& Blandford 1991). A nonthermal, red source could, however, be subject to strong, achromatic microlensing, and if this is happening, our model would definitely be invalid. High-accuracy photometric monitoring may determine whether microlensing is at work. (In a different approach to modeling this source, Kayser et al. 1990 invoke microlensing and ignore the measured magnifications in deriving a macrolensing model. They present two quite different models that fit the image locations; one involves a single galaxy with eccentricity larger than 0.9 , while the other uses two galaxies. Neither model is compatible with our $\mathrm{CO}$ data [see below].)

Our model is able to reproduce both the image locations and the relative fluxes to within the measurement accuracies. The best-fitting parameters are $b=(0.088)^{2}, s=0.21, \gamma_{c}=$ $0.09, \gamma_{s}=0.15, q=0.61, \gamma_{1}=-0.05, \gamma_{2}=-0.08$. The lensing galaxy is centered at $(-0.13,+0.56)$ relative to image A. The corresponding ellipticity in the surface density contours is $\epsilon=0.3$, and the position angle of its major axis is $125^{\circ}$ (see Fig. 2a). This model is quite different from the single-galaxy model of Kayser et al. (1990). It is roughly orthogonal in orientation and much less elliptical. The total point-source magnification $\mu=220$ is much larger than the Kayser et al. model, implying that the source is correspondingly less powerful. It also implies that the time delays are very much shorter. Adopting $z_{d}=\Omega_{0}=1$, for example, we obtain $t_{\mathrm{BA}}=$ $-0.3, t_{\mathrm{CA}}=-0.5, t_{\mathrm{DA}}=0.9 \mathrm{~h}^{-1}$ day, which will be a challenge to measure. We have preferred this model because it seems most able to accommodate the $\mathrm{CO}$ data. However, as the foregoing discussion implies, it is not unique, and much more 
will need to be known before this source becomes a good candidate for determining the Hubble constant.

We now adopt this lens model and use it to derive $\mathrm{CO}$ source models that provide the best fit to the red and the blue images. Using the adopted lens model, we were able to produce an excellent fit to the contours of the redshifted $\mathrm{CO}$ image; the fit to the blueshifted image was less good, but was acceptable granted the resolution of the observation (Figs. $2 b$ and $2 c$ ). We find that the peaks of the red and the blue source are displaced by $\sim 0$. $15 \equiv 550 h^{-1}$ pc with respect to the quasar, roughly on opposite sides of the optical source. This suggests that the molecular gas orbits the quasar. We obtain a dynamical estimate of the central mass of $\sim 10^{10} h^{-1} M_{\odot}$, adopting a low inclination $i \sim 30^{\circ}$, on the grounds that $\mathrm{H} 1413+117$ is a broad absorption line (BAL) quasar. (Note, though, that the quasar is not being observed through the molecular gas as the high extinction $A_{v}>1000$ would render it optically invisible.) The fact that each of the multiply imaged CO sources, each with $\sim 7 \sigma$ in significance, maps back to a single image on the opposite side of the quasar gives us additional confidence in our modeling and the reality of the observed differences between the red and blue images. The two $\mathrm{CO}$ sources are not exactly symmetric about the quasar, and this may reflect a real asymmetry in the gas distribution, an error in relative astrometry between optical and CO images $(\lesssim 0$ ". 2$)$, or an inaccuracy in the lens model.

The overall CO magnification depends on the source size (Eisenhardt et al. 1996). For our predicted overall CO source size, we estimate that the mean magnification is $\mu_{\mathrm{CO}} \sim 10$. A similar magnification is probably appropriate for the farinfrared emission discussed below. Adopting this value, the associated molecular gas mass is calculated from the observed flux $\left(S_{\mathrm{CO}(7-6)}=41 \mathrm{Jy} \mathrm{km} \mathrm{s}^{-1}\right)$ to be $\sim 2 \times 10^{10} h^{-2} M_{\odot}$ using a standard Galactic CO-to- $\mathrm{H}_{2}$ conversion factor (Young \& Scoville 1991; Solomon, Downes, \& Radford 1992). A lower than solar metallicity requires a somewhat larger gas mass to account for the observed CO flux. Because of the small source size $\left(\lesssim 3^{\prime \prime}\right)$, our interferometer measurement should include all the emission; the existing single-dish measurement of $\mathrm{CO}$ (7-6) flux is quite uncertain because it suffers from lack of a good spectral baseline (R. Barvainis 1996, private communication). This inferred mass of molecular gas is comparable to the upper bound derived on the basis of dynamics, and we therefore suppose that it is a fair estimate of the total molecular hydrogen mass. This in turn implies that molecular gas may dominate the mass distribution on the $\sim 1 \mathrm{kpc}$ scale just as in the ultraluminous infrared galaxies in the local universe (Scoville \& Soifer 1991; Yun \& Scoville 1995; Bryant \& Scoville 1996).

\section{PHYSICAL PROPERTIES OF THE MOLECULAR GAS SURROUNDING THE QUASAR}

The CO-emitting gas appears to be both warm and dense. The peak observed brightness temperature for the $\mathrm{CO}$ (7-6) line seen in the individual $32 \mathrm{MHz}$ channel maps is $2.2 \mathrm{~K}$ above the brightness temperature of the cosmic background radiation. Gravitational lensing preserves brightness temperature, and this excess corresponds to a Planck brightness temperature at the source redshift of $24 \mathrm{~K}$. If the lensed image fills $\frac{1}{2}\left(\frac{1}{4}\right)$ of the synthesized beam, then the inferred temperature of the gas is about 50 (100) K. The CO (7-6) line is bright, and the near-unity $(\sim 0.9)$ inferred brightness temperature ratio of our $\mathrm{CO}(7-6)$ measurement to that of the CO (3-2) measurement by Barvainis et al. (1994) suggests that the CO-emitting gas is thermalized and optically thick if both transitions originate from the same region. (In support of this, we note that both transitions have the same line width and profile.) Because the $\mathrm{CO} J=$ 7 rotational level lies $155 \mathrm{~K}$ above the ground state, producing significant CO (7-6) emission and the observed line ratio requires a minimum density of $n>10^{5} \mathrm{~cm}^{-3}$ and an excitation temperature $T_{\mathrm{ex}} \gtrsim 100 \mathrm{~K}$.

The inferred dust temperature from the continuum spectrum is $\sim 100 \mathrm{~K}$ (Barvainis et al. 1992; Downes et al. 1992; Rowan-Robinson et al. 1993), and this is consistent with the expectation that the dust and gas should be in thermal equilibrium at the density required for producing the observed CO (7-6) flux. The observed spectral energy distribution for the infrared and submillimeter emission can be interpreted as thermal emission from dust with total mass of $\sim 5 \times 10^{7} M_{\odot}$, which corresponds to a gas mass $2.5 \times 10^{10} M_{\odot}$ after correcting for the lens magnification and adopting a gas-to-dust ratio $\sim 500$ appropriate to infrared galaxies in the local universe (Sanders, Scoville, \& Soifer 1991). This is in good agreement with the gas mass estimate from the $\mathrm{CO}$ emission above, although large uncertainties are associated with both estimates.

The ratio of the infrared luminosity to the molecular gas $L_{\mathrm{FIR}} / M_{\mathrm{H}_{2}}$ is a crude measure of the efficiency of converting gas into radiant energy either through stars or via an active nucleus. Among the most luminous infrared galaxies found in the local universe, this ratio approaches $\sim 100 L_{\odot} / M_{\odot}$. However, for $\mathrm{H} 1413+117$ and FSC $10214+4724$, the observed ratios exceed $200 L_{\odot} / M_{\odot}$ (neglecting the possibility of different magnification for the infrared and $\mathrm{CO}$ emission). This suggests that the bulk of the large infrared luminosities of both of these objects is provided by a quasar, which is hidden in the case of FSC $10214+4724$. In these discussions it is assumed that the $\mathrm{CO}$-emitting region coincides with the infrared-emitting region because a large amount of dust associated with the CO-emitting dense molecular gas would dominate the total infrared emission.

In summary, we note that the molecular gas complex in $\mathrm{H} 1413+117$ is similar in size, mass, density, and temperature to that observed in the nuclei of local infrared galaxies (Sanders et al. 1991). This is consistent with theoretical models in which a large quantity of gas driven into the nuclear region by mergers or interactions (Barnes \& Hernquist 1991) is processed by starbursts over a timescale $\sim 10^{8} \mathrm{yr}$, so that it builds up a high metallicity (Scoville \& Soifer 1991). This process may account for the strong $\mathrm{CO}$ emission from the ISM of this $z=2.6$ system observed when the universe was only a few billion years old.

Here we have also demonstrated that gravitational lens indeed can be a valuable tool for probing objects at cosmological distances by yielding details that are be normally inaccessible for the current generation of observational instruments (Zwicky 1937). For example, the resolution of the distribution and kinematics of the massive molecular gas complex surrounding a similarly distant unlensed quasar would require a tenfold improvement both in resolution and sensitivity over the existing instruments. Over 20 gravitation- 
ally lensed objects suitable for detailed studies using this gravitational lens telescope are now known in the literature (see Blandford \& Narayan 1992; Maoz et al. 1993; King \& Browne 1996), and studies exploiting this magnifying characteristic at all wavelengths may be rewarded with valuable new insights on these distant sources.
The authors are grateful to E. Falco for kindly providing the HST image for the analysis and comparison, and to $\mathrm{R}$. Barvainis for sharing his unpublished data. This manuscript also benefited greatly from the careful reading and useful suggestions by P. Ho and referee R. Antonucci. This research is supported in part by NSF grant AST 93-14079.

\section{REFERENCES}

Barnes, J. E., \& Hernquist, L. E. 1991, ApJ, 370, L65

Barvainis, R., Antonucci, R., \& Coleman, P. 1992, ApJ, 399, L19

Barvainis, R., Antonucci, R., Hurt, T., Coleman, P., \& Reuter, H.-P. 1995, ApJ, 451, L9

Barvainis, R., Tacconi, L. R., Antonucci, R., Alloin, D., \& Coleman, P. 1994, Nature, 371, 586

Blandford, R. D., \& Narayan, R. 1992, ARA\&A, 30, 311

Bryant, P. M., \& Scoville, N. Z. 1996, ApJ, 457, 678

Downes, D., Radford, S. J .E., Greve, A., Thum, C., Solomon, P. M., \& Wink, J. E. 1992, ApJ, 398, L25

Eisenhardt, P. R., Armus, L., Hogg, D. W., Soifer, B. T., Neugebauer, G., \& Werner, M. W. 1996, ApJ, 461, 72

Falco, E. E. 1993, in Gravitational Lenses in the Universe, ed. J. Surdej et al. (Liège: Université de Liège), 127

Hewitt, J. N., Turner, E. L., Schneider, D. P., Burke, B. F., Langston, G. I., \& Lawrence, C. R. 1988, Nature, 333, 537

Kayser, R., Surdej, J., Condon, J. J., Kellermann, K. I., Magain, P., Remy, M., \& Smette, A. 1990, ApJ, 364, 15

King, L. J., \& Browne, I. W. A. 1996, MNRAS, 282, 67
Magain, P., Surdej, J., Swings, J.-P., Borgeest, U., Kayser, R., Kuhr, H., Refsdal, S., \& Remy, M. 1988, Nature, 334, 325

Maoz, D., et al. 1993, ApJ, 409, 28

Rauch, K. P. \& Blandford, R. D. 1991, ApJ, 381, L39

Rowan-Robinson, M., et al. 1993, MNRAS, 261, 513

Sanders, D. B, Scoville, N. Z., \& Soifer, B. T. 1991, ApJ, 370, 158

Schneider, P., Ehlers, J., \& Falco, E. E. 1992, Gravitational Lenses (Berlin: Springer)

Scoville, N. Z., Carlstrom, J. C., Chandler, C. J., Phillips, J. A., Scott, S. L. Tilanus, R. P., \& Wang, Z. 1992, PASP, 105, 1482

Scoville, N. Z., \& Soifer, B. T. 1991, in Massive Stars and Starburst, ed. K Leitherer (Cambridge: Cambridge Univ. Press), 233

Shepherd, M. C., Pearson, T. J., \& Taylor, G. B. 1994, BAAS, 26, 987

Solomon, P. M., Downes, D., \& Radford, S. J. E. 1992, ApJ, 387, L55

Wilner, D. J., Zhao, J.-H., \& Ho, P. T. P. 1995, ApJ, 453, L91

Young, J. S., \& Scoville, N. Z. 1991, ARA\&A, 29, 581

Yun, M. S., \& Scoville, N. Z. 1995, ApJ, 451, L45

Zwicky, F. 1937, Phys. Rev., 51, 290 